\title{
Mesoporous Silica Nanoparticles with a Core-Shell Structure for Drug Delivery \\ Yufang Zhu*
}

School of Materials Science and Engineering, University of Shanghai for Science and Technology, 516 Jungong Road, Shanghai, 200093, People's Republic of China

Mesoporous silica nanoparticles (MSNs) have some unique advantages including high surface area and large pore volume, tunable particle size $(10-1000 \mathrm{~nm})$ and pore diameter $(2-30 \mathrm{~nm})$, uniform mesoporosity, flexible morphology, facile surface functionalization, excellent biocompatibility and biodegradation. On the one hand, textural properties of MSNs provide the possibility to load high amount of drugs within MSNs carriers. On the other hand, there are abundant silanol groups on the surfaces of mesoporous channels and the outer surfaces of MSNs, which facilitate the surface functionalization to allow for a better control over the drug diffusion kinetics. In addition, functional materials, such as magnetic nanoparticles, luminescent materials and polymers, can be combined with MSNs to form functional MSNs, which induce MSNs as multifunctional platforms to realize the targeted controlled drug delivery and/or imaging. Therefore, since the report on MCM-41 mesoporous silica for drug delivery by M. Vallet-Regí group in 2001 [1], MSNs have been considered to be excellent candidates as carriers for drug delivery [2-5].

Recently, many efforts have been made in the structure design and functional optimization to advance the development of mesoporous silica-based drug delivery systems [6], such as embedding of functional materials in mesoporous channels, core-shell structure and functionalization on the surface of mesoporous silica. Among them, the core-shell structured functional MSNs can combine new functions in the platform without blocking the mesoporous channels. Therefore, the core-shell structured functional MSNs are desirable for drug delivery, and have been one of the most active research topics on the applications of MSNs.

To increase the drug loading of a carrier is one of main approaches for enhancing therapeutic efficacy. Conventional MSNs can load a dose of therapeutic drug with 200-300 mg (maximally about $600 \mathrm{mg}$ ) drug per $1 \mathrm{~g}$ silica [7]. However, hollow MSNs, also called MSNs with hollow core-mesoporous shell structure, are able to achieve a superhigh drug loading capacity due to the hollow cores providing more space to load drugs [8], typically more than $1 \mathrm{~g}$ drug per $1 \mathrm{~g}$ silica [9]. In cancer chemotherapy, such a high drug loading capacity is in great favor of high chemotherapeutic efficacy, particularly against the multi-drug resistance (MDR) in tumor, because an inefficient intracellular accumulation of chemotherapeutic drug frequently leads to chemotherapeutic failure, or even to the evocation of the MDR in tumors.

Because pure MSNs can not realize the targeted drug delivery, and can not track/evaluate the efficiency of drug release in disease diagnosis and therapy, the combination with MSNs and functional materials to form the core-shell structure is a smart strategy to solve the limitations, especially to form magnetic and/or luminescent mesoporous silica nanoparticles. Thus, these MSNs with a core-shell structure can be as multifunctional platforms for simultaneous targeted drug delivery, fast diagnosis, and efficient therapy.

Among the magnetic MSNs, the core-shell structured nanoparticles with magnetic core and mesoporous silica shell are considered as the most important and desirable structure for drug delivery combined with targeting and/or hyperthermia functions [10-12]. Especially for magnetic MSNs with rattle-type structure, their interstitial spaces between mesoporous silica shell and magnetic cores are beneficial for high drug loading capacity $[11,12]$.

Luminescent labeling is a real-time, simple, and effective way to monitor the route of drug-transport carriers in a living system. Drug delivery systems with luminescent labels can easily evaluate the efficiency of the drug release and disease therapy. The luminescent labels, such as organic dyes, quantum dots (QDs) and rare-earth (RE)doped phosphors, can be embedded in or coated by mesoporous silica to form a core-shell structured luminescent MSNs [13-15], which reduce the interference of the environment on the luminescent property of the luminescent core and completely utilize the excellent advantages of mesoporous silica shell. Furthermore, the combination of magnetism and luminescence in one MSN provides a new generation MSNs carrier with a broad range of functionalities. The magnetic-luminescent MSNs can serve as an all-in-one diagnostic and therapeutic tool, which could be used to visualize and simultaneously treat various diseases.

Except for the magnetic and/or luminescent MSNs, other functional MSNs also have been intensively developed for drug delivery. The $\mathrm{Au}$ core-mesoporous silica shell structured MSNs combine the photothermal characteristic of Au nanorods with the mesoporosity of MSNs in one body [16], which are favorable for cancer treatment due to the combination of hyperthermia with the chemotherapeutic drugs by synergistic effect. On the other hand, a variety of functional coreshell MSNs with mesoporous silica cores and functional shells have also been developed for stimuli-responsive controlled drug delivery [17]. That is to say, mesoporous silica cores serve as containers for drugs and functional shells serve as "gatekeepers" to trigger drug release only upon exposure to stimuli, which could decrease side-effect to protect the healthy organs from toxic drugs and prevent the decomposition/ denaturing of the drugs before reaching the targeted organs or tissues.

To date, multifunctional drug delivery systems based on the coreshell structured MSNs have been designed and optimized in order to deliver the drugs into the targeted organs or cells, with a controllable release fashion by virtue of various internal and external triggers. The systems will be able to track the released drug molecules in a living system. Therefore, these developments are encouraging and

*Corresponding author: Yufang Zhu, School of Materials Science and Engineering University of Shanghai for Science and Technology, 516 Jungong Road, Shanghai, 200093, People's Republic of China, E-mail: zjf2412@163.com

Received July 19, 2013; Accepted July 20, 2013; Published July 23, 2013

Citation: Zhu Y (2013) Mesoporous Silica Nanoparticles with a Core-Shell Structure for Drug Delivery. J Bioanal Biomed 5: e117. doi:10.4172/1948-593X.1000e117

Copyright: ( $) 2013$ Zhu Y. This is an open-access article distributed under the terms of the Creative Commons Attribution License, which permits unrestricted use, distribution, and reproduction in any medium, provided the original author and source are credited. 
show great promise in biomedical applications. However, there are still many challenges need to be overcome and investigated more comprehensively and thoroughly for these functional MSNs to advance its biological and biomedical applications. MSNs can transport through the cell membranes and deliver drugs into cells, but there are still many questions to be answered for future practical applications, such as the pharmacokinetics and pharmacodynamics of drugs loaded in MSNs, biodistribution, the acute and chronic toxicities, long-term in vivo degradation and compatibility of MSNs [18]. On the other hand, the developed synthesis methods to functional MSNs with a core-shell structure are limited to produce a small amount of nanoparticles. Therefore, the scaled synthesis routes to functional MSNs with a coreshell structure are very important for the final possible biomedical applications.

\section{References}

1. Vallet-RegíM, Rámila A, del Real R P, Pérez-Pariente J (2001) A new property of MCM-41: drug delivery system. Chem Mater 13: 308-311.

2. Yang P, Gai S, Lin J (2012) Functionalized mesoporous silica materials for controlled drug delivery. Chem Soc Rev 41: 3679-3698.

3. Tang FQ, Li LL, Chen D (2012) Mesoporous silica nanoparticles: synthesis, biocompatibility and drug delivery. Adv Mater 24: 1504-1534.

4. He QJ, Shi JL (2011) Mesoporous silica nanoparticle based nano drug delivery systems: synthesis, controlled drug release and delivery, pharmacokinetics and biocompatibility. J Mater Chem 21: 5845-5855.

5. Manzano M, Vallet-Regí M (2010) New developments in ordered mesoporous materials for drug delivery. J Mater Chem 20: 5593-5604.

6. Li Z, Barnes JC, Bosoy A, Stoddartbc JF, Zink JI (2012) Mesoporous silica nanoparticles in biomedical applications. Chem Soc Rev 41: 2590-2605.

7. He QJ, Shi JL, Chen F, Zhu M, Zhang LX (2010) An anticancer drug delivery system based on surfactant-templatedmesoporous silica nanoparticles. Biomaterials 31: 3335-3346.
8. Chen Y, Chen HR, Guo LM, He QJ, Chen F, et al. (2010) Hollow/rattle-type mesoporous nanostructures by a structural difference-based selective etching strategy. ACS Nano 4: 529-539.

9. Zhu YF, Shi JL, Shen WH, Chen HR, Dong XP, et al. (2005) Preparation of novel hollow mesoporous silica spheres and their sustained-release property. Nanotechnology16: 2633-2638.

10. Kim J, Kim HS, Lee N, Kim T, Kim H, et al. (2008) Multifunctional uniform nanoparticles composed of a Magnetite nanocrystal core and a mesoporous silica shell for magnetic resonance and fluorescence imaging and for drug delivery. Angew Chem Int Ed 47: 8438-8441.

11. Wu H, Liu G, Zhang S, Shi J, Zhang L, et al. (2011) Biocompatibility, MR imaging and targeted drug delivery of a rattle-type magnetic mesoporous silica nanosphere system conjugated with PEG and cancer-cell-specific ligands. J Mater Chem21: 3037-3045.

12. Zhu Y F, Ikoma T, Hanagata N, Kaskel S (2010) Rattle-type $\mathrm{Fe}_{3} \mathrm{O}_{4} @ \mathrm{SiO}_{2}$ hollow mesoporous spheres as carriers for drug delivery.Small 6: 471-478.

13. Lei J, Wang L, Zhang J (2011) Superbright multifluorescent core-shell mesoporousnanospheres as trackable transport carrier for drug. ACS Nano 5: 3447-3455

14. Hu X, Zrazhevskiy P, Gao X (2009) Encapsulation of single quantum dots with mesoporous silica. Ann Biomed Eng37: 1960-1966.

15. Kang X, Cheng Z, Li C, Yang D, Shang M, et al. (2011) Core-shell structured up-conversion luminescent and mesoporous $\mathrm{NaYF}_{4}: \mathrm{Yb}^{3+} / \mathrm{Er}^{3+} @ \mathrm{nSiO} @$ $\mathrm{mSiO}_{2}$ nanospheres as carriers for drug delivery. J Phys Chem C 115: 15801 15811.

16. Xie L, Dong B, Jiang Z, Wang Y, Liu T, et al. (2011) Synthesis of novel coreshell structural AuNR@MCM-41 die infrared light-driven release of drug. J Mater Res 26: 2414-2419.

17. Coll C, Mondragón L, Martínez-Máńez R, Sancenón F, Dolores Marcos M, et al. (2011) Enzyme-mediated controlled release systems by anchoring peptide sequences on mesoporous silica supports . Angew Chem Int Ed50: 2138-2140.

18. Chen Y, Chen HR, Shi JL (2013) In vivo bio-safety evaluations and diagnostic/therapeutic applications of chemically designed mesoporous silica nanoparticles. Adv Mater 25: 3144-3176. 